\title{
Indication or diagnosis should be required on prescriptions
}

Michael T Rupp, PhD, FAPhA; Terri L Warholak, PhD, RPh, CPHQ, FAPhA; and Anita C Murcko, MD, FACP, FAMIA

\section{SUMMARY}

Although prospective drug utilization review and patient counseling have long been recognized as professional and ethical responsibilities of pharmacists, the implementation of the Omnibus Budget Reconciliation Act of 1990 made them legal responsibilities. Ensuring the safety and effectiveness of prescription pharmaceutical care requires that all members of the prescriber-patient-pharmacist triad are equally informed about the therapeutic plan for which the pharmacist is professionally, ethically, and legally responsible for properly implementing. Providing pharmacists with the clinical indication or diagnosis is an important and long overdue first step.
A provision of the 21st Century Cures Act passed in 2016 and implemented April 5, 2021, now provides patients with free and unfettered electronic access to their personal health care information including, but not limited to, physical exam notes, imaging, laboratory and pathology reports, progress notes, consultations, and discharge summaries. ${ }^{1,2} \quad$ Although this "open notes" mandate received pushback from some quarters, it has generally been hailed as a positive step toward a more inclusive and participatory approach to health care by patients and providers. ${ }^{3}$

While we applaud the effort to provide patients with better access to their health care information, it is discouraging that pharmacists, the health professionals who prescribers rely on to implement their medication plans for patients, do not have similar access to decision-relevant patient information. Instead, dispensing pharmacists, especially those in retail settings such as community, mail, and specialty pharmacies, continue to practice in a virtual information vacuum with respect to the clinical information that prescribers considered when they created their therapeutic plan for the patient.

\section{The Pharmacist's Responsibility in Drug Therapy}

A bit of historical context is helpful to understand why lack of access by pharmacists to patient information is a barrier to effective care and a threat to patient safety. The prospective drug utilization review (pro-DUR) provisions of the Omnibus Budget Reconciliation Act of 1990 (OBRA '90) required pharmacists to evaluate prescribed medication therapy before dispensing to ensure that it is appropriate, medically necessary, and not likely to result in adverse events. ${ }^{4}$ Following the act's implementation in 1993, these pro-DUR provisions were written into every state pharmacy practice act, making them a legally mandated responsibility of pharmacists, as was the requirement that

\section{Author affiliations}

Michael T Rupp, PhD, FAPhA, Midwestern University College of Pharmacy-Glendale Campus, Glendale, AZ; Terri L Warholak, $\mathrm{PhD}, \mathrm{RPh}, \mathrm{CPHQ}, \mathrm{FAPhA}$, University of Arizona College of Pharmacy, Tucson; and Anita C Murcko, MD, FACP, FAMIA, College of Health Solutions, Arizona State University, Tempe.

AUTHOR CORRESPONDENCE:

Michael T Rupp, 623.572.3528;

mtrupp@midwestern.edu

J Manag Care Spec Pharm. 2021;27(8):1136-39

Copyright $\odot 2021$, Academy of Managed Care Pharmacy. All rights reserved.

pharmacists offer to counsel patients on their therapy.

While the expectations for pharmacist review of prescribed medication therapy were significantly increased by OBRA ' 90 , the information inputs available to help make these critical clinical judgments were not. Two particularly relevant questions that a pharmacist must ask and answer to fulfill the pro-DUR requirements of OBRA-90 are "what is the problem being treated?" and "what is the clinical objective of the therapy?". Answering both questions is essential if a pharmacist is to accurately assess the appropriateness of the prescribed medication therapy and adequately counsel the patient. 
Fulfilling their professional responsibilities is difficult enough given the paucity of patient information to which pharmacists routinely have access in many practice settings. When adding the potential complexity introduced by off-label prescribing, it becomes virtually impossible to do so with any confidence. ${ }^{5}$ Lacking the diagnosis or clinical indication for which the medication is being prescribed, pharmacists must infer it from knowledge of the drug. This inference, however, is subject to error.

\section{Benefits of Indication-Based Prescribing and Dispensing}

Asserting in their 2016 perspective published in the New England Journal of Medicine that it is "time to enter the age of reason," Schiff et al presented a cogent argument for universal adoption of indication-based prescribing, ${ }^{6}$ noting that "there are 5 'rights' required for safe medication ordering and use: the right patient, the right drug, the right dose, the right time, and the right route. But there's a sixth element that must be correct-and we believe it's time to add to each prescription an ingredient that's currently conspicuously missing: the right indication."' Among the benefits cited in their commentary were improved medication safety, enhanced patient education and empowerment, improved clinical documentation, and better communication with all members of the health care team, specifically including pharmacists.

Research has demonstrated that including indication on electronic prescriptions reduces prescribing errors and the frequency with which pharmacists are required to consult prescribers to clarify or correct prescription orders, ${ }^{7,8}$ thereby reducing workflow interruptions for pharmacists and prescribers. ${ }^{9}$ Providing indications on prescriptions has also been shown to improve the quality of patient counseling, leading to better medication adherence..$^{10,11}$ Pharmacists have also been found to make significantly better pro-DUR-related clinical decisions when they have access to the diagnosis or the reason for use of a prescribed medication..$^{12}$ An additional benefit is that it would allow pharmacy benefit managers to conduct prior authorization more efficiently and with fewer disruptions in care. ${ }^{13}$

In recognition of these benefits, pharmacy professional associations and medication safety organizations have been calling for the use of diagnosis, indication, or reason on medication orders for over 2 decades. In 1997, the National Coordinating Council for Medication Error Reporting and Prevention stated that "prescription orders should include a brief notation of purpose (eg, for cough), unless considered inappropriate by the prescriber."14 In 1993, the American
Pharmaceutical Association (now the American Pharmacists Association) provided additional support when it adopted a resolution stating that "APhA supports a legal requirement for the provision of diagnosis, lab results, and/or intended therapeutic outcome to accompany prescription orders." ${ }^{15}$ This resolution was restated in 2001 that "APhA supports the inclusion of the diagnosis or indication for use for which the medication is ordered." ${ }^{15}$ In 2004, the National Association of Boards of Pharmacy added their endorsement by adopting a resolution to "encourage national and state medical associations and other interested parties to support legislative and regulatory efforts in the states to require prescribers to include the indication for the medication on all prescriptions and medication orders issued orally, in writing, or transmitted electronically."16 More recently, the Institute for Safe Medication Practices, the premier medication safety organization in the United States, also reiterated and reinforced its own long-standing support for indication-based prescribing and communication of the indication to the pharmacist. ${ }^{17}$

The authors of this commentary include 2 researchers in medication safety management with first-hand experience practicing pharmacy in an information-rich environment (Indian Health Service). The third author is a board-certified internal medicine physician with over 2 decades of experience in private practice. We have each observed the effect of enhanced access to information on improved patient outcomes from the research bench and the patient bedside.

\section{Potential Barriers}

If there is such a broad-based and long-standing support for inclusion of the indication or diagnosis on prescriptions, why has it still not happened? Why have state boards of pharmacy (BOPs) not exercised their discretionary rule-making power to add this as a requirement for a legal prescription order? The answer, we believe, is the same reason why most BOPs have still not declared handwritten prescription orders illegal outside of legitimate emergency situations, despite the indisputable fact that doing so would immediately and significantly improve patient safety: they simply lack the political courage to do it.

The unwillingness of BOPs to issue such a rule may be due at least in part to the opposition it is likely to generate from state medical associations and organizations representing other prescriber groups. It must be recalled that BOP members are gubernatorial political appointees in most states and are therefore subject to political considerations. Few governors are likely to appreciate 
being placed in the awkward position between citizen safety and powerful interest groups.

Beyond political considerations, BOPs likely also recognize that some of the reasons for opposition to such a proposed rule may have merit. In our own state of Arizona, we have conducted a number of surveys of prescribers to identify their concerns relative to such a potential requirement. ${ }^{18}$ Among the most commonly cited reasons for opposition were increased time required of the prescriber, patient confidentiality or privacy concerns, fears that the information may be used by payers to deny coverage for nonformulary drugs or off-label uses, and skepticism regarding the ability of software to accurately transmit and display the information.

The fear that a required indication or diagnosis might be used by payers to deny coverage for some prescriptions appears to be a particular concern to some prescribers, especially since selected off-label uses are widely considered to be the standard of care for some medications. But while requiring indication or diagnosis may result in the need for prescribers to provide additional justification in some cases, having this information universally associated with the medication order could assist in the generation of realworld evidence to support eventual labeling changes to recognize additional indications. Moreover, such information would be essential in a possible future of indication-based drug pricing in which different prices might be charged for different uses of a particular drug, thereby better linking the price of a drug to its value in use. ${ }^{19}$

Another legitimate concern expressed by prescribers is the concern that they may not always be in a position to indicate a definitive diagnosis or clinical indication, since the patient's response to the medication may be part of a differential diagnosis. In such cases, unequivocal disambiguation of alternative indications is not possible, and the implementation of such a requirement would have to allow for such circumstances.

Beyond prescribers, we can envision that there may be resistance to such a requirement from pharmacy itself. It is not difficult to imagine that some pharmacists and pharmacy corporations may oppose such a requirement due to concerns of increasing dispensing time and cost, reduced productivity, and/or increased liability exposure.

Pharmacy computer systems would have to be able to receive and accurately display this information at the point of dispensing, which may require software updates for some pharmacies with concurrent cost implications. Moreover, efficiently and effectively using this additional information may require some additional clinical skills development of pharmacy staff that would also have cost implications.

We recognize that concerns about requiring indication or diagnosis on prescription orders are not without merit. However, we believe these concerns, while valid, are far outweighed by the benefits to safer and more effective patient care that results from a more complete exchange of information between prescriber and pharmacist. While access to the complete medical record would be ideal and should be the long-term goal, providing the pharmacist with the clinical indication or diagnosis is an essential move in the right direction.

\section{Conclusions}

Implementation of the "open notes" provisions of the 21st Century Cures Act will provide patients with access to virtually all of their medical records. Although pro-DUR and patient counseling have long been recognized as professional and ethical responsibilities of pharmacists, the implementation of OBRA '90 made them legal responsibilities. Ensuring the safety and effectiveness of prescription pharmaceutical care requires that all members of the prescriber-patient-pharmacist triad are equally informed about the therapeutic plan for which the pharmacist is responsible for properly implementing on behalf of the prescriber. Providing pharmacists with the clinical indication or diagnosis is an important first step that is long overdue.

\section{DISCLOSURES}

No funding was received for the writing of this article. Warholak has received grant funding through the University of Arizona from Sinfonia Rx, Pharmacy Quality Alliance, and the Arizona Department of Health Services, unrelated to this work. The other authors have nothing to disclose.

\section{REFERENCES}

1. 21st Century Cures Act: Interoperability, Information Blocking, and the ONC Health IT Certification Program. 85 FR 2564225961. May 1, 2020. Accessed November 9, 2020. https://www.federalregister. gov/documents/2020/05/01/202007419/21st-century-cures-actinteroperability-information-blockingand-the-onc-health-it-certification

2. Open Notes. Federal rules mandating open notes. October 30, 2020. Accessed November 13, 2020. https://www.opennotes.org/ onc-federal-rule-interoperabilty-information-blocking-and-open-notes/

3. Johnson CK. More US patients have easy, free access to doctor's notes. AP News. November 1, 2020. Accessed November 9, 2020. https://apnews.com/ article/technology-virus-outbreakdc762ec0fd60f363adc8e5d4ce42b7ff 
4. Omnibus Budget Reconciliation Act of 1990 (OBRA-90). Pub L No. 101-508, 104 Stat. 1388, (1990). Accessed May 26, 2021. https://www.govinfo.gov/content/pkg/ STATUTE-104/pdf/STATUTE-104-Pg1388. pdf

5. Rupp MT. The pharmacist's role in off-label prescribing. Arch Intern Med. 2010;170:658.

6. Schiff GD, Seoane-Vazquez E, Wright A. Incorporating indications into medication ordering-time to enter the age of reason. N Engl J Med. 2016;375(4):306-09.

7. Galanter WL, Falck S, Burns ML, et al. Indication-based prescribing prevents wrong-patient medication errors in computerized order entry (CPOE). J Am Med Inform Assoc. 2013;20(3):477-81.

8. Galanter WL, Bryson ML, Falck S, et al. Indication alerts intercept drug name confusion errors during computerized entry of medication orders. PLoS One. 2014;9(7):e101977.

9. Warholak TL, Rupp MT, Leal S, et al. Assessing the effect of providing a pharmacist with patient diagnosis on electronic prescription orders: a pilot study. Res Social Adm Pharm. 2014;10(1):246-51.

10. Teichman PG, Caffee AE. Prescription writing to maximize patient safety. Fam Pract Manag. 2002;9(7):27-30.
11. Kuntz JL, Safford MM, Singh JA, et al. Patient-centered interventions to improve medication management and adherence: a qualitative review of research findings. Patient Educ Couns. 2014;97(3):310-26.

12. Warholak-Juarez T, Rupp MT, Salazar TA, Foster S. Effect of patient information on the quality of pharmacists' drug use review decisions. J Am Pharm Assoc (Wash). 2000;40(4):500-08.

13. Forrester C. Benefits of prior authorizations. J Manag Care Spec Pharm. 2020;26(7):820-22. doi: 10.18553/ jmcp.2020.26.7.820

14. National Coordinating Council for Medication Error Reporting and Prevention. Recommendations to enhance accuracy of prescription/ medication order writing. Adopted September 4, 1996. Revised October 22, 2014. Accessed November 10, 2020. https://www.nccmerp.org/recommendations-enhance-accuracy-prescription-writing\#: :text=Prescription\%20 orders $\% 20$ should $\% 20$ include $\% 20$ a,prescribing\%20and\%20dispensing\%20 a\%20medication

15. American Pharmacists Association. APhA-ASP adopted resolutions: 19732019. Updated October 10, 2019. Accessed May 26, 2021. https://www.pharmacist. com/Portals/0/PDFS/ASP/APhAASP\%20Adopted\%20Resolutions\%20 -\%20Updated\%20October\%202019. pdf?ver=JhD URYnaNpLayhsYzvmQ\%3D\%3D
16. National Association of Boards of Pharmacy. Resolution 100-7-04: Medication Indication on the Prescription. Newsletter. Vol. 24, No. 8. September 2005. Page 159. Accessed May 26, 2021. https://nabp.pharmacy/wp-content/ uploads/2016/07/Sept05NABP.pdf

17. Institute for Safe Medication Practices. Is an indication-based prescribing system in our future? November 17, 2016. Accessed November 5, 2020. https:// www.ismp.org/resources/indicationbased-prescribing-system-our-future

18. Loera C, Olsen J, So A, Murata J, et al. Prescriber and pharmacist attitudes toward inclusion of diagnosis or clinical indication on prescription orders. J Am Pharm Assoc (2003). 2021;S15443191(20)30636-1. doi: 10.1016/j. japh.2020.12.015

19. Towse A, Cole A, Zamora B. The debate on indication-based pricing in the U.S. and five major European countries. Office of Health Economics Consulting. May 2018. Accessed February 23, 2021. https://www.ohe.org/publications/ debate-indication-based-pricing-us-andfive-major-european-countries\# 